\title{
Calcium Activity of Upper Thoracic Dorsal Root Ganglion Neurons in Zucker Diabetic Fatty Rats
}

\author{
Marie Louise Ghorbani, ${ }^{1}$ Niels C. B. Nyborg, ${ }^{2}$ Bjarne Fjalland, ${ }^{1}$ and Majid Sheykhzade ${ }^{1}$ \\ ${ }^{1}$ Department of Drug Design and Pharmacology, Faculty of Health and Medical Sciences, University of Copenhagen, \\ 2100 Copenhagen, Denmark \\ ${ }^{2}$ Non-Clinical Development, Novo Nordisk A/S, 2760 Maaloev, Denmark
}

Correspondence should be addressed to Marie Louise Ghorbani; mlmuus@yahoo.dk

Received 14 December 2012; Revised 19 February 2013; Accepted 20 February 2013

Academic Editor: Gunnar Gislason

Copyright (C) 2013 Marie Louise Ghorbani et al. This is an open access article distributed under the Creative Commons Attribution License, which permits unrestricted use, distribution, and reproduction in any medium, provided the original work is properly cited.

\begin{abstract}
The aim of the present study was to examine the calcium activity of $\mathrm{C}_{8}-\mathrm{T}_{5}$ dorsal root ganglion (DRG) neurons from Zucker diabetic fatty rats. In total, 8 diabetic ZDF fatty animals and 8 age-matched control ZDF lean rats were employed in the study. $C_{8}-T_{5}$ dorsal root ganglia were isolated bilaterally from 14 to 18 weeks old rats, and a primary culture was prepared. Calcium activity was measured ratiometrically using the fluorescent $\mathrm{Ca}^{2+}$-indicator Fura-2 acetoxymethyl ester. All neurons were stimulated twice with $20 \mathrm{mM} \mathrm{K}$, followed by stimulation with either 0.3 or $0.5 \mu \mathrm{M}$ Capsaicin, alone or in combination with algogenic chemicals (bradykinin, serotonin, prostaglandin E2 (all $10^{-5} \mathrm{M}$ ), and adenosine $\left(10^{-3} \mathrm{M}\right)$ ) at $\mathrm{pH} 7.4$ and 6.0. Neurons from diabetic animals exhibited an overall increased response to stimulation with $20 \mathrm{mM} \mathrm{K}^{+}$compared to neurons from control. Stimulation with Capsaicin alone caused an augmented response in neurons from diabetic animals compared to control animals. When stimulated with a combination of Capsaicin and algogenic chemicals, no differences between the two groups of neurons were measured, neither at $\mathrm{pH} 7.4$ nor 6.0. In conclusion, diabetes-induced alterations in calcium activity of the DRG neurons were found, potentially indicating altered neuronal responses during myocardial ischemia.
\end{abstract}

\section{Introduction}

It is estimated that 366 million people worldwide in the age of 20-79 years were affected by diabetes in 2010 and the prevalence is expected to increase to 552 million people in 2030 [1]. Cardiovascular disease is the main cause of death among people with diabetes, accounting for $50 \%$ of all diabetic deaths [1]. Diabetes mellitus is per se a risk factor for developing cardiovascular disease, with ischemic heart disease being the most frequently found [2], but also diabetic neuropathy, specifically cardiovascular autonomic neuropathy, is considered a risk factor [3-6]. Silent myocardial ischemia and infarction as well as sudden death are also more frequently found in patients where diabetes and cardiovascular autonomic neuropathy coexist and cardiovascular autonomic neuropathy is furthermore believed to be a contributing factor to the higher mortality and morbidity found in patients with diabetes mellitus following myocardial ischemia or infarction [3-7].
Neurons that can contribute to the pathological picture of autonomic neuropathy are the afferent sensory nerves coming from the organ, the neurons involved in central processing of the nociceptive signal, and the efferent autonomic nerves leading to the organ and regulating its function [8].

During myocardial ischemia, lactic acid is produced, causing a decrease in local $\mathrm{pH}$ (decrease of epicardial $\mathrm{pH}$ from 7.35 to 6.98 after $5 \mathrm{~min}$ of coronary artery occlusion has been demonstrated) [9], and release of inflammatory and/or algogenic (i.e., pain-causing) mediators, such as bradykinin, adenosine, serotonin, prostaglandin $\mathrm{E}_{2}$, and histamine [10]. These metabolic components have been shown to activate/sensitize spinal and vagal afferents in the heart. The effect is (1) release of neuropeptides leading to local effects in the myocardium and (2) transmission of the nociceptive signal through the primary afferents (via the dorsal root ganglia (DRGs)) to segments $\mathrm{C}_{8}-\mathrm{T}_{9}$ in the spinal cord [11-15] or to the nucleus of the solitary tract in medulla oblongata. Neurons 
can project to the brain giving rise to perception of anginal pain and associated feelings (e.g., nausea and anxiety) and/or activate autonomic efferent fibers, leading to regulation of cardiovascular function [11].

Diabetes-induced alterations in cardiac sensory nerves could thus have pronounced effects on the way a patient responds to myocardial ischemia and infarction, including changes in perception of the pain often accompanying myocardial ischemia and infarction, and in the cardiac compensatory responses.

Diabetic neuropathy is thought to be caused by several hyperglycemia-induced changes: neurovascular dysfunction, lack of trophic support, and metabolic changes, such as an increased polyol pathway flux, increased production of advanced glycation end products (AGEs), and oxidative stress [16]. Furthermore, alterations in $\left[\mathrm{Ca}^{2+}\right]_{i}$ homeostasis have been suggested to contribute to the pathogenesis of diabetic neuropathy [16-18]. Altered calcium channel activity has been shown in primary [18] and secondary sensory neurons from diabetic rodents [19].

Very little is known of the consequences of diabetes on sensory nerve function of primary afferents innervating the heart. Schultz found a decreased activity of cardiac vagal afferents in STZ-diabetic rats compared to control rats after epicardial application of bradykinin and capsaicin [20] and recently, diabetes-induced alterations in the spinal processing of nociceptive input from the heart were found in diabetic rats following cardiac stimulation [21].

The purpose of the present study was to investigate the calcium activity of $\mathrm{C}_{8}-\mathrm{T}_{5}$ dorsal root ganglion (DRG) neurons (primary neuron) in Zucker diabetic fatty rats, a spontaneous animal model for type 2 diabetes, and the effect of stimulation with algogenic substances, known to play part during myocardial ischemia, as well as with capsaicin, the pungent ingredient in chili peppers, at $\mathrm{pH} 7.4$ and 6.0. To the best of our knowledge, this is the first study assessing the calcium activity in upper thoracic DRG neurons from any type of diabetic rat.

\section{Methods and Materials}

2.1. Animals. Animal procedures were approved by the Danish Animal Inspectorate (license no. 2004/561-943).

Experiments were performed in male diabetic Zucker diabetic fatty (ZDF) rats as well as age-matched nondiabetic ZDF rats. The ZDF rat is a spontaneous animal model for type 2 diabetes in which the animals are homozygous for nonfunctional leptin receptors [22, 23]. On a Purina 5008 diet, hyperglycemia will develop as early as an age of approximately 7 weeks [23].

The animals were stabled in pairs and were fed with Purina 5008, an energy-rich fodder. Once a week, the animals were weighed and blood glucose was measured from a tail vein using a glucometer (OneTouch Ultra2, Lifescan).

2.2. Primary Culture. At the age of $14-18$ weeks, the rats were decapitated and $\mathrm{C}_{8}-\mathrm{T}_{5}$ DRG's were removed bilaterally and placed in Hank's Buffered Salt Solution (HBSS).
The ganglia were transferred to $5 \mathrm{~mL}$ papain solution ( $2 \mathrm{mg} / \mathrm{mL}$, Sigma-Aldrich) for $35 \mathrm{~min}$ at $37^{\circ} \mathrm{C}$. After centrifugation $(2800 \mathrm{rpm}, 2 \mathrm{~min}$ ) the supernatant was removed and the pellet was resuspended in $5 \mathrm{~mL}$ of a collagenase/dispase solution (Collagenase: $1.11 \mathrm{mg} / \mathrm{mL}$, Medinova; Dispase: $1.33 \mathrm{mg} / \mathrm{mL}$, Roche Diagnostics $\mathrm{GmbH}$ ). The solution was kept at $37^{\circ} \mathrm{C}$ for $20 \mathrm{~min}$ with routinely mixing. After centrifugation (2800 rpm, $2 \mathrm{~min}$ ) and removal of supernatant, the cells were triturated in $1 \mathrm{~mL}$ HBSS (by at least 10 passages through a fire-polished Pasteur pipette) and gently placed on $7 \mathrm{~mL}$ percoll (20\% v/v, Sigma-Aldrich). After centrifugation (2800 rpm, $7 \mathrm{~min}$ ), the percoll containing ganglia remnants was removed and the pellet resuspended in $1 \mathrm{~mL}$ complete F-12 medium. After centrifugation $(2800 \mathrm{rpm}, 3 \mathrm{~min})$, the supernatant was removed leaving approximately $30-60 \mu \mathrm{L}$ of fluid. The cells were resuspended in the remaining fluid and approximately $10-20 \mu \mathrm{L}$ of the cell suspension was placed on each of three poly-d-lysine/laminin $12 \mathrm{~mm}$ coverslips (Becton Dickinson CDC). The coverslips were placed at $37^{\circ} \mathrm{C}$ for two hours to allow the cells to adhere to the coverslips and afterwards, $2.5 \mathrm{~mL}$ of complete F-12 medium was added to each well. The cells were kept at $37^{\circ} \mathrm{C}$ until use (2-12 hours).

2.3. Calcium Imaging. The cells were transferred to PSS, adjusted to $\mathrm{pH} 7.4$ and loaded with the fluorescent $\mathrm{Ca}^{2+}$ indicator Fura-2 acetoxymethyl ester (Fura-2/AM, $4.4 \mu \mathrm{M}$ ) dissolved in DMSO and Chremaphor EL detergent $(4: 1)$ for $30-60 \mathrm{~min}$ at $37^{\circ} \mathrm{C}$. After loading, the cells were transferred to fresh PSS buffer and allowed to stand for approx. $30 \mathrm{~min}$ in order to ensure completion of the cytoplasmatic dye deesterification [24].

Changes in intracellular calcium concentrations in neurons from $\mathrm{C}_{8}-\mathrm{T}_{5}$ DRG's were measured by FURA-2 ratiometric imaging in the acutely dissociated culture. At the time of measurement, cells were round and without processes.

The cells were illuminated alternately with light of $340 \mathrm{~nm}$ (specific for $\mathrm{Ca}^{2+}$-bound Fura-2) and $380 \mathrm{~nm}$ (specific for $\mathrm{Ca}^{2+}$-free Fura-2), and light of $510 \mathrm{~nm}$ was collected via a cooled CCD camera (Hamamatsu). The photomultiplier was coupled to a computer for data acquisition.

2.4. Experimental Protocols. Six different protocols were employed in the study, only one protocol per coverslip. Each coverslip was initially stimulated twice with $20 \mathrm{mM} \mathrm{K}^{+}$for $10 \mathrm{~s}$ separated by $7 \mathrm{~min}$ wash with PSS. The first depolarization was undertaken to ensure vitality of the neurons and saturation of the intracellular compartments and was excluded from further analysis. After washout of the second administration of $\mathrm{K}^{+}$, neurons were stimulated for $10 \mathrm{~s}$ with one of the following solutions: (1) $0.3 \mu \mathrm{M}$ capsaicin, (2) $0.5 \mu \mathrm{M}$ capsaicin, (3) mixture of algogenic chemicals (AC) combined with $0.3 \mu \mathrm{M}$ capsaicin, (4) AC combined with $0.5 \mu \mathrm{M}$ capsaicin, (5) AC combined with $0.3 \mu \mathrm{M}$ capsaicin at $\mathrm{pH} 6.0$, or (6) AC combined with $0.5 \mu \mathrm{M}$ capsaicin at $\mathrm{pH}$ 6.0. After stimulation with one of these solutions, the neurons were washed with PSS for $8 \mathrm{~min}$. The experiment was concluded with a third $\mathrm{K}^{+}$ stimulation to evaluate the continued viability of the neuron for the extent of the protocol. The mixture of algogenic 
chemicals contained bradykinin, serotonin, prostaglandin $\mathrm{E}_{2}$, all in the concentration of $10^{-5} \mathrm{M}$, and adenosine $\left(10^{-3} \mathrm{M}\right)$. All substances used for stimulation were obtained from SigmaAldrich.

$\mathrm{pH} 6.0$ was chosen for the two acidic protocols studies as DRG neurons containing TRPV1 and acid sensing ion channels (ASICs) have been demonstrated to react to this $\mathrm{pH}$ [25-27].

2.5. Analysis. Prior to experiments, inclusion criteria for data analysis were defined: (1) labeling with Fura-2, (2) only neurons included, (3) neurons should stay within its marked area throughout the experiments, (4) cells responding to $20 \mathrm{mM} \mathrm{K}^{+}$were included ( $\geq 20 \%$ increase from baseline), and (5) only neurons returning to baseline during washout period were included.

To monitor the $\left[\mathrm{Ca}^{2+}\right]_{i}$ changes in single cells, specific areas of interest were chosen for cells and for background. For each time frame, the ratio of background-corrected fluorescence intensities detected at $510 \mathrm{~nm}$ and resulting from excitation at 340 and $380 \mathrm{~nm}$ was calculated:

$$
R=\frac{I_{340}-I_{340}(\text { background })}{I_{380}-I_{380}(\text { background })} .
$$

This ratio is an arbitrary value and was used as a measure of the activity level and to record changes in the activity level of the neurons.

Baseline, peak value, and AUC of light intensity ratios were measured for each response in Chart 5 (AD Instruments). For $\mathrm{K}^{+}$stimulations, AUC was calculated from $0 \mathrm{~s}$ to $180 \mathrm{~s}$ after initiation of response $\left(\mathrm{AUC}_{0-180 \mathrm{~s}}\right)$; for all other stimulations, $\mathrm{AUC}_{0-60 \mathrm{~s}}$ was calculated, where $0 \mathrm{~s}$ indicates beginning of rise in $\left[\mathrm{Ca}^{2+}\right]_{i}$. The maximum increase from baseline, that is, the peak height, was calculated for all neurons, both in absolute terms $(\Delta)$, and relative as the percentage increase from baseline $(\%-\Delta)$. For all stimulations except $\mathrm{K}^{+}, \Delta, \%-\Delta$ and AUC were furthermore compared to the $\mathrm{K}^{+}$response by calculating the ratio of a given response and the preceding $\mathrm{K}^{+}$response for each neuron.

Neuron diameter was measured and the neurons were classified as small (S, $<30 \mu \mathrm{m})$, medium (M, 30-39 $\mu \mathrm{m})$, or large $(\mathrm{L}, \geq 40 \mu \mathrm{m})$, similar to the classification by $\mathrm{Lu}$ et al. [28]. To further analyze the distribution of neuron diameter in control and diabetic animals, the neurons were divided into bins, each of $5 \mu \mathrm{m}$ width.

Cells responding to $20 \mathrm{mM} \mathrm{K}^{+}$were included in the analysis as neurons [18]. Responsiveness was defined as $\geq 20 \%$ increase from baseline (i.e., $\%-\Delta \geq 20 \%$ ), after stimulation with any substance in the protocol.

During analysis it was found that neurons stimulated with 0.3 and $0.5 \mu \mathrm{M}$ Capsaicin elicited similar responses, and this was also seen for neurons stimulated with the combination of AC and either 0.3 or $0.5 \mu \mathrm{M}$ Capsaicin. For subsequent analysis, 0.3 and $0.5 \mu \mathrm{M}$ Capsaicin was therefore combined and henceforward is referred to as "Capsaicin" and furthermore, the protocols of $\mathrm{AC}$ in combination with either 0.3 or $0.5 \mu \mathrm{M}$ Capsaicin were combined and henceforward are referred to "AC + Capsaicin."

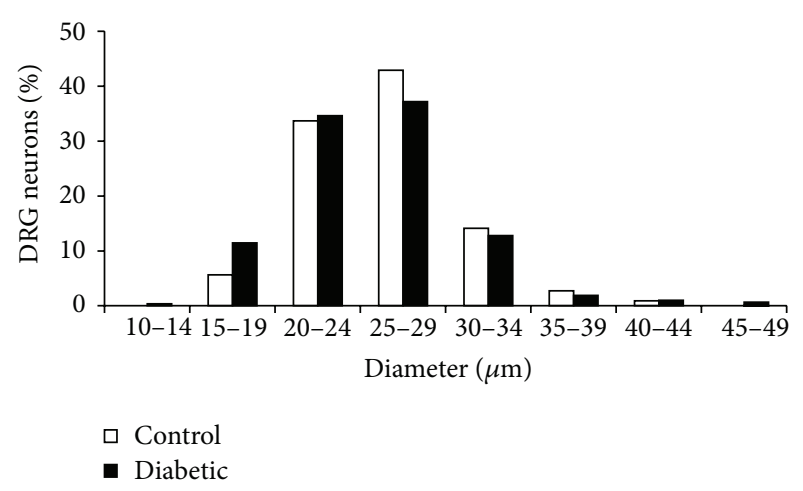

FIGURE 1: Distribution of neuronal cell diameter. The neurons were divided into $5 \mu \mathrm{m}$ bins. Small-diameter neurons had diameters < $30 \mu \mathrm{m}$, medium-sized neurons had diameters between 30-39 $\mu \mathrm{m}$, and large-diameter neurons had diameters of $\geq 40 \mu \mathrm{m}$. The majority of tested neurons were small-sized. $n($ control $)=552, n$ (diabetic $)=$ 586.

2.6. Statistical Analysis. The results are presented either as mean \pm SEM or as median (5th percentile; 95th percentile), depending on normality of data. Comparisons were made with Student's $t$-test or, when appropriate, the nonparametric Mann-Whitney Rank Sum Test. Comparisons of diameter distribution were done using Chi-square test. Statistical tests were performed with SigmaStat 3.11 and GraphPad Prism 5. $P<0.05$ was used as level of significance.

\section{Results}

3.1. Animals. In total, 8 diabetic ZDF animals and 8 agematched control ZDF rats were used in this study. At the age of 7 weeks, the ZDF diabetic rats had developed significantly higher blood glucose concentrations than control rats and this hyperglycemia persisted for the whole experimental period. At the time of experiment, all diabetic animals had an elevated blood glucose level of $27.5 \pm 4.5 \mathrm{mM}$ compared to $6.2 \pm 0.5 \mathrm{mM}$ in control animals $(P<0.001, n=16)$. The body weight was larger in diabetic animals from 7 weeks of age and this increased body weight persisted throughout week 14 after which body weight no longer differed between control and diabetic animals. At the time of experiment, body weight was $378.0 \pm 26.3 \mathrm{~g}$ for the diabetic animals and $365 \pm$ $23.9 \mathrm{~g}$ for the control animals $(P>0.05, n=16)$.

3.2. Neurons. The mean cell diameter of the recorded DRG neurons was slightly smaller for diabetic than for control animals $(25 \mu \mathrm{m}(18.0 ; 33.2)$ versus $26 \mu \mathrm{m}(19.0 ; 33.0), P<0.05$, $n=1124)$.

The distribution of small-, medium-, and large-sized neurons, though, did not differ between the two animal groups; for neurons from diabetic animals, $82 \%$ were smallsized, $17 \%$ medium-sized, and $1 \%$ large-sized compared to $83 \%, 15 \%$, and $2 \%$, respectively, for neurons from control animals. The distribution based on neuron diameter was also not significantly different in control and diabetic animals (Figure 1). When differentiating, however, between neurons 


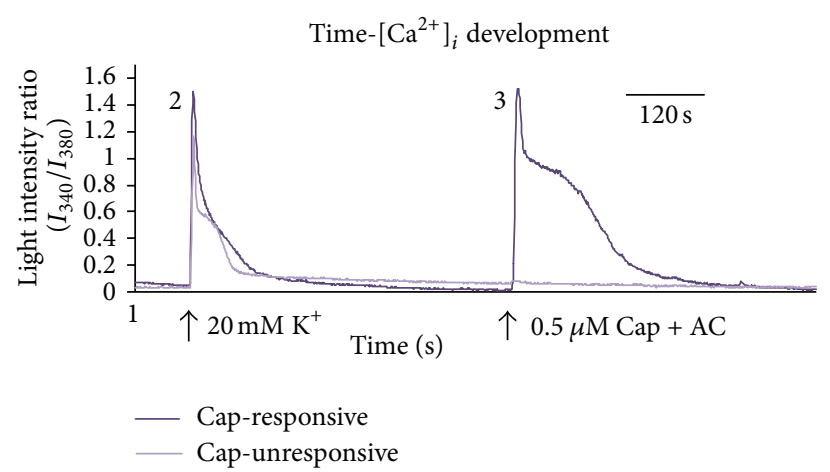

(a)

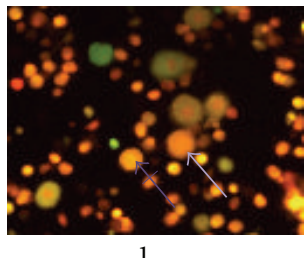

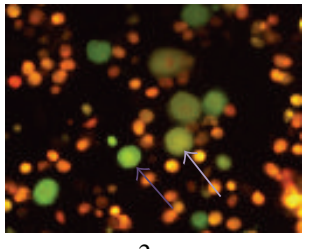

2

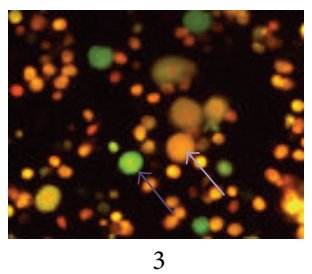

(b)

FIGURE 2: Example of calcium activity measurement. The figure depicts the trace of two cells obtained during a single experiment: a capsaicinresponsive (dark purple) and a capsaicin-unresponsive (light purple) neuron. The three small photomicrographs show the same neurons, at different stages of the experiment: (1) before stimulation, (2) during stimulation with $20 \mathrm{mM} \mathrm{K}^{+}$, and (3) during stimulation with $0.5 \mu \mathrm{M}$ Capsaicin + algogenic chemicals. Black arrows beneath the trace indicate the beginning of stimulation. Time scale bar; $120 \mathrm{~s}$. Cap; capsaicin.

TABLE 1: Quantitative response of all neurons to $10 \mathrm{~s}$ stimulation with $20 \mathrm{mM} \mathrm{K}{ }^{+}$. Results are shown as median (5\%-95\% percentile).

\begin{tabular}{|c|c|c|c|c|}
\hline Animal & $n$ & $\mathrm{AUC}_{0-180 \mathrm{~s}}$ & $\Delta$ & $\%-\Delta$ \\
\hline Control & 549 & $30.00(3.04-110.11)$ & $0.92(0.25-1.47)$ & $156.36(41.77-248.25)$ \\
\hline Diabetic & 578 & $41.41(3.89-121.72)^{*}$ & $1.08(0.31-1.72)^{*}$ & $182.26(35.86-308.52)^{*}$ \\
\hline
\end{tabular}

AUC: Area under the curve for $0-180 \mathrm{~s}, \Delta$ : the absolute change in $\left[\mathrm{Ca}^{2+}\right]_{i}$ (fluorescence ratio), \%- $\Delta$ : the change in $\left[\mathrm{Ca}^{2+}\right]_{i}$ (fluorescence ratio) measured in $\%$ change from baseline. ${ }^{*} P<0.001$ versus control neurons.

responding to either of the capsaicin protocols (capsaicinresponsive) and neurons not responding to the tested capsaicin protocol (capsaicin-unresponsive), analysis showed that the distribution of "capsaicin-responsive" neurons from diabetic animals was significantly different from the distribution of control animals $(P<0.05)$ with a median neuron diameter in diabetic animals of $23 \mu \mathrm{m}(16.7 ; 30.0)$ compared to $25 \mu \mathrm{m}(18.9 ; 34.1)$ in control animals $(P<0.01)$.

The baseline $\mathrm{Ca}^{2+}$ level did not differ between diabetic and control animals $(0.58(0.46 ; 0.80)$ versus 0.59 (0.47; $0.77), P>0.05, n=1127)$. Response to a given stimulus was elicited by an increase in intracellular $\mathrm{Ca}^{2+}$ level and viewed as an increase in fluorescence intensity ratio, that is, a peak that slowly returned to baseline. An example of a capsaicin-responsive and-unresponsive neuron following stimulation with $20 \mathrm{mM} \mathrm{K}^{+}$and a capsaicincontaining stimulus is depicted in Figure 2. Neuron responses with and without shoulder were found among both groups of animals as well as among capsaicin-responsive and unresponsive neurons and thus, presence or lack of shoulder in the response was not confined to single groups of neurons investigated.
3.3. $K^{+}$-Induced Depolarization. Control as well as diabetic neurons responded to stimulation with $20 \mathrm{mM} \mathrm{K}^{+}$by an increase in $\left[\mathrm{Ca}^{2+}\right]_{i}$, assessed by an increase in $340 / 380 \mathrm{~nm}$ fluorescence ratio. The intracellular $\mathrm{Ca}^{2+}$ level returned to baseline during the washing period. A representative trace of the time- $\left[\mathrm{Ca}^{2+}\right]_{i}$ relationship is shown in Figure 3. The diabetic neurons showed an overall increased responsiveness to stimulation with $20 \mathrm{mM} \mathrm{K}{ }^{+}$compared to neurons from control animals (Table 1). This was seen as an augmented AUC, peak height $(\Delta)$, and $\%$ increase from baseline $(\%-\Delta)$. The increased responsiveness was observed both in "capsaicinresponsive" and "capsaicin-unresponsive" neurons (Table 2). For both control and diabetic animals it was seen that capsaicin-responsive neurons generally had a higher responsiveness to $20 \mathrm{mM} \mathrm{K}^{+}$than capsaicin-unresponsive neurons (Table 2).

3.4. Capsaicin Protocols. Stimulation with capsaicin alone, 0.3 or $0.5 \mu \mathrm{M}$ (no combination) caused a rise in $\left[\mathrm{Ca}^{2+}\right]_{i}$ for approximately $23 \%$ of the neurons in both groups of animals. The neurons from diabetic animals exhibited an augmented response, assessed by a larger peak height, 
TABLE 2: Quantitative response to $10 \mathrm{~s}$ stimulation with $20 \mathrm{mM} \mathrm{K}$. The results were subcategorized according to whether they responded to the capsaicin protocol the respective neuron was stimulated with. Results are shown as median (5\%-95\% percentile).

\begin{tabular}{|c|c|c|c|c|c|}
\hline Animal & Capsaicin response & $n$ & $\mathrm{AUC}_{0-180 \mathrm{~s}}$ & $\Delta$ & $\%-\Delta$ \\
\hline \multirow{2}{*}{ Control } & $\mathrm{Y}$ & 157 & $34.68(3.05-138.27)$ & $1.05(0.27-1.65)$ & $175.36(39.50-290.96)$ \\
\hline & $\mathrm{N}$ & 392 & $28.02(3.02-93.19)^{\#}$ & $0.88(0.25-1.30)^{\#}$ & $149.64(42.24-233.10)^{\#}$ \\
\hline \multirow{2}{*}{ Diabetic } & $\mathrm{Y}$ & 193 & $42.97(5.08-155.09)$ & $1.25(0.38-1.96)^{*}$ & $202.78(60.60-353.75)^{*}$ \\
\hline & $\mathrm{N}$ & 385 & $40.99(3.45-105.17)^{* \$}$ & $1.01(0.28-1.59)^{* \#}$ & $170.37(21.97-288.22)^{* \#}$ \\
\hline
\end{tabular}

AUC: Area under the curve for $0-180 \mathrm{~s}, \Delta$ : the absolute change in $\left[\mathrm{Ca}^{2+}\right]_{i}$ (fluorescence ratio), \%- $\Delta$ : the change in $\left[\mathrm{Ca}^{2+}\right]_{i}$ (fluorescence ratio) measured in $\%$ change from baseline. Y: Yes and N: No. ${ }^{*} P<0.001$ versus corresponding control neurons, ${ }^{\#} P<0.001$ and ${ }^{\$} P<0.05$ versus corresponding capsaicin-responsive neurons.

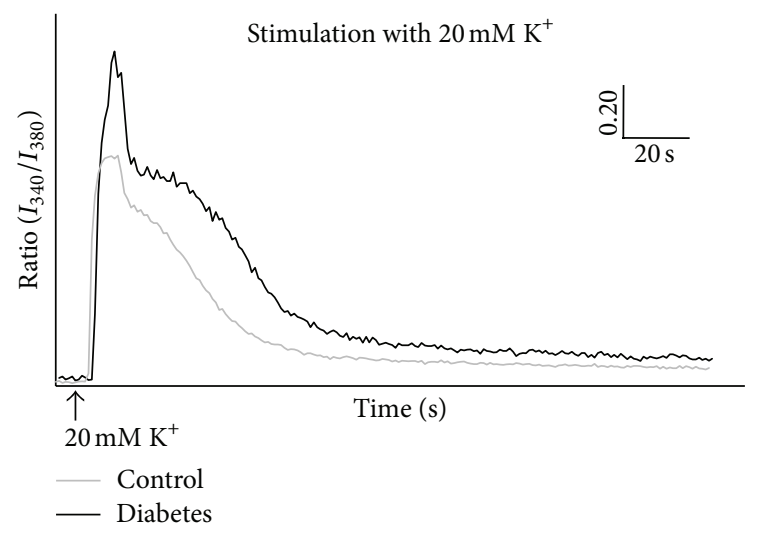

FIGURE 3: Example of time- $\left[\mathrm{Ca}^{2+}\right]_{i}$ ratio development in dorsal root ganglion neurons from control and diabetic rats. The neurons were stimulated with $20 \mathrm{mM} \mathrm{K}$. For clarity in the illustration, the traces were background corrected. Arbitrary scale; vertical scale: $0.20(340 / 380 \mathrm{~nm}$ light intensity ratio), and horizontal scale: $20 \mathrm{~s}$.

percentage increase from baseline, and AUC (Figures 4(a)4(c) and Table 3).

Capsaicin-responsive neurons stimulated with capsaicin in combination with the algogenic substances at either $\mathrm{pH}$ 7.4 or 6.0 were followed by increases in $\left[\mathrm{Ca}^{2+}\right]_{i}$ in neurons from both diabetic and control animals (Figures 4(d), 4(g), and $4(\mathrm{j}))$. None of the stimulations gave rise to differences in peak height or AUC between diabetic and control animals (Figures $4(\mathrm{~d})-4(\mathrm{k})$, Table 3 ).

Scatter plots of the capsaicin responses indicated that neurons responding to capsaicin-containing solutions might be divided in high- and low-responders (Figure 5).

Stimulation with $0.5 \mu \mathrm{M}$ capsaicin in combination with the algogenic chemicals at $\mathrm{pH} 6.0$ caused a significantly augmented $\mathrm{Ca}^{2+}$ response compared to any of the other stimulations. This was seen for neurons from diabetic animals $(\Delta ; 1.63$ (0.16; 1.87), AUC; 63.51 (2.87; 97.95), $P<0.001$ compared to every other stimulation of diabetic neurons, $n=63)$ as well as from control animals $(\Delta ; 1.7(0.21 ; 2.37)$; AUC; $72.13(1.58 ; 117.24), P<0.001$ compared to every other stimulation of control neurons, $n=13)$. The responses were similar in neurons from control and diabetic animals $(P>$ 0.05).

\section{Discussion}

The major findings of the present study were the following: (1) baseline $\left[\mathrm{Ca}^{2+}\right]_{i}$ were unaltered in $\mathrm{C}_{8}-\mathrm{T}_{5}$ DRG neurons from ZDF diabetic compared to control animals, (2) $\mathrm{K}^{+}$-induced and (3) capsaicin-induced depolarization caused augmented $\left[\mathrm{Ca}^{2+}\right]_{i}$ currents in neurons from diabetic animals.

4.1. Baseline $\mathrm{Ca}^{2+}$-Levels. The current study showed no differences in baseline $\left[\mathrm{Ca}^{2+}\right]_{i}$ in $\mathrm{C}_{8}-\mathrm{T}_{5}$ DRG neurons from diabetic and control animals. This is in accordance with several other studies performed on DRG neurons from STZdiabetic mice and rats, $\mathrm{db} / \mathrm{db}$ diabetic mice and $\mathrm{BB} / \mathrm{W}$ rats $[18,29-32]$, and on dorsal horn neurons from lumbar $\mathrm{L}_{6}$ $\mathrm{L}_{7}$ spinal cord segments from STZ-diabetic rats $[19,33]$. Shutov et al. [34], however, have found an increased baseline $\left[\mathrm{Ca}^{2+}\right]_{i}$ in $\mathrm{L}_{4}-\mathrm{L}_{6}$ DRG neurons from STZ-induced diabetic rats at the same time as an unaltered baseline in $\mathrm{DH}$ neurons, suggesting a differential effect by diabetes on primary and secondary neurons [34]. The segmental location of the neurons investigated also appear to be affected differently by diabetes as neurons with longer axons are more susceptible to neuropathic changes than neurons with shorter axons [8]. This is supported by reports of an increase in baseline $\left[\mathrm{Ca}^{2+}\right]_{i}$ in STZ-diabetic DRG neurons extracted from $\mathrm{L}_{4}-\mathrm{L}_{6}$ levels where the neurons have longer axons because they innervate the hind paws $[18,34]$, while Huang and colleagues, in the same study, found no baseline changes in $\mathrm{C}_{5}-\mathrm{L}_{3}$ DRG neurons [18], which agrees with the results from the present study on $\mathrm{C}_{8}-\mathrm{T}_{5}$ DRG neurons.

4.2. $\mathrm{K}^{+}$-Induced Depolarization. The present study found an increased depolarization-induced $\left[\mathrm{Ca}^{2+}\right]_{i}$ current in DRG neurons from ZDF diabetic animals compared to control animals. The increase comprised an absolute and a percentage increase in amplitude from baseline $\left[\mathrm{Ca}^{2+}\right]_{i}$ and an increased $\mathrm{AUC}_{(0-180 \mathrm{~s})}$. Hall et al. also found an increased elevation of $\left[\mathrm{Ca}^{2+}\right]_{i}$ in thoracic DRG neurons (d. $20-40 \mu \mathrm{m}$ ) after stimulation with $50 \mathrm{mM} \mathrm{KCl}$ for $6 \mathrm{~s}$ in $\mathrm{BB} / \mathrm{W}$ rats [32]. Most other studies with DRG and DH neurons have not detected differences in the amplitude of $\left[\mathrm{Ca}^{2+}\right]_{i}$ elevation between diabetic and nondiabetic rats and mice [19, 29-31, 33-35]. An exception, however, is Huang et al. who found a decreased amplitude of depolarization-induced $\left[\mathrm{Ca}^{2+}\right]_{i}$ current in small DRG neurons from $\mathrm{L}_{4}-\mathrm{L}_{6}$ in STZ-diabetic animals (14-week 


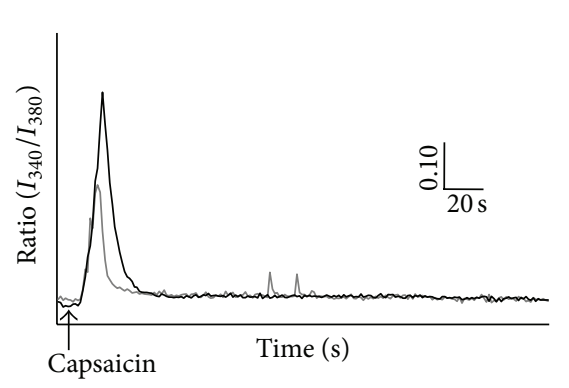

(a)

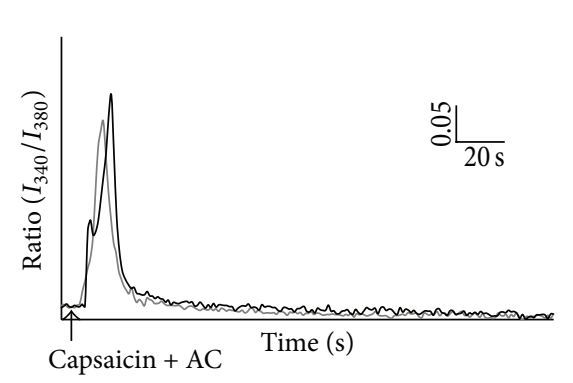

(d)

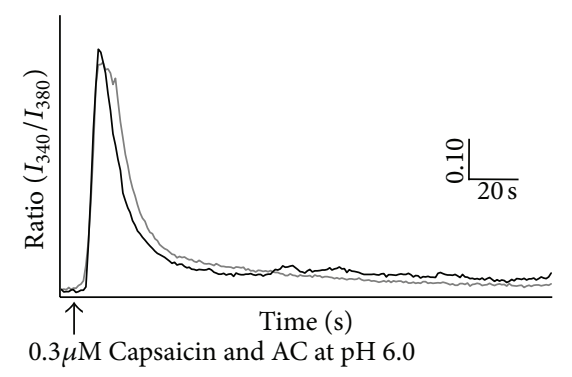

(g)

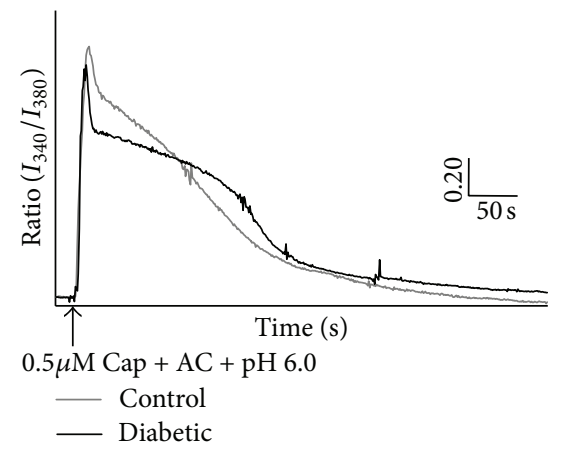

(j)

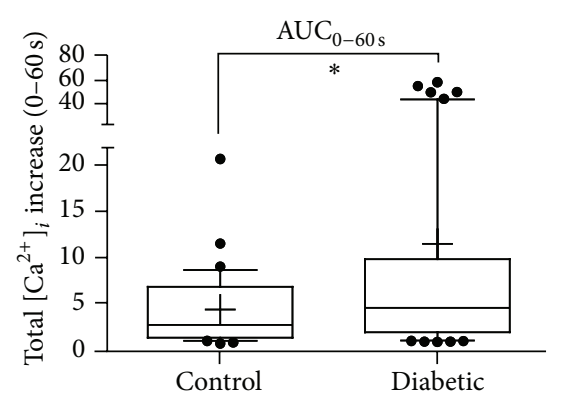

(b)

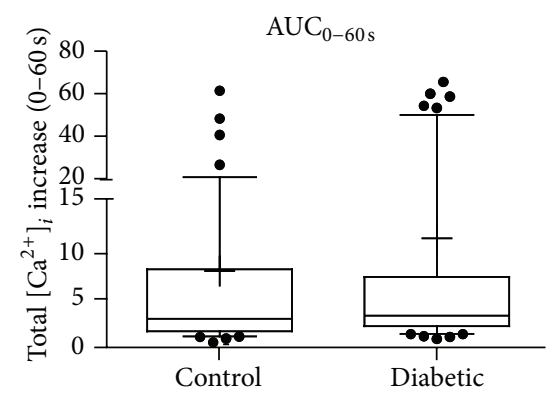

(e)

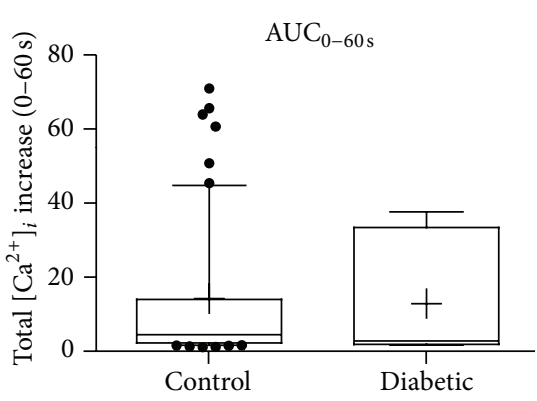

(h)

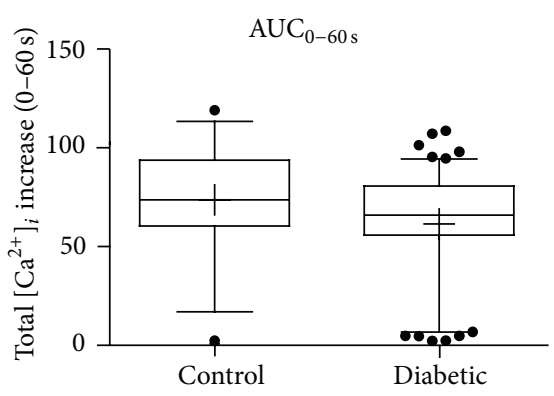

$(\mathrm{k})$

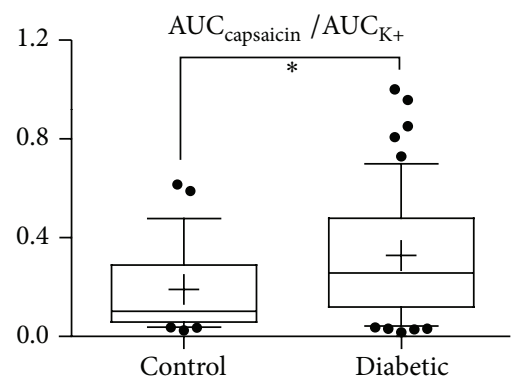

(c)

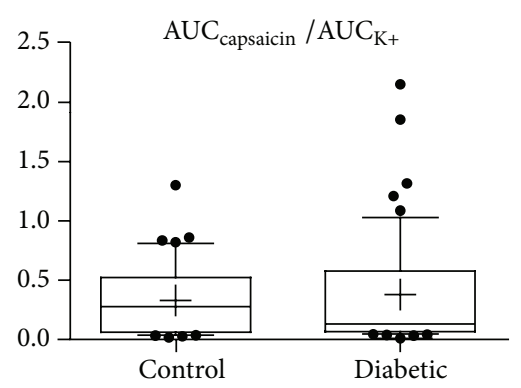

(f)

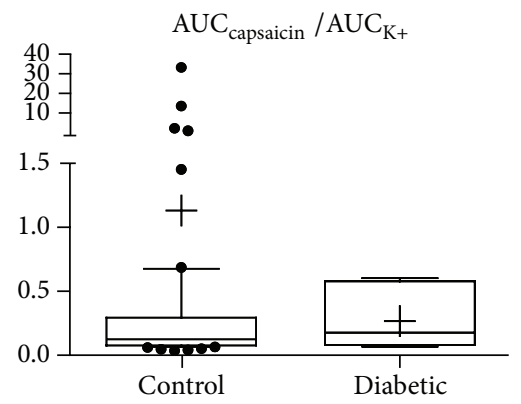

(i)

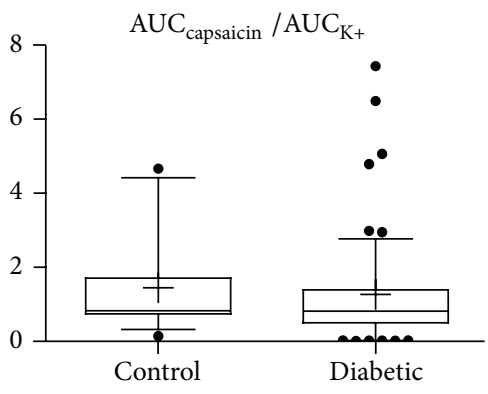

(l)

FIGURE 4: Traces and box plots from capsaicin experiments. Panels (a), (d), (g), and (j) show traces (examples) of the time-[Ca $\left.{ }^{2+}\right]_{i}$ development for control and diabetic dorsal root ganglion neurons. The arrows indicate the onset of $10 \mathrm{sec}$ stimulation with (a) capsaicin, (d) combination of Capsaicin and algogenic chemicals (AC); (g) combination of $0.3 \mu \mathrm{M}$ Capsaicin and $\mathrm{AC}$ at pH 6.0, and (j) $0.5 \mu \mathrm{M} \mathrm{Capsaicin} \mathrm{and} \mathrm{AC}$ at pH 6.0. The traces were background corrected. As a measure of $\left[\mathrm{Ca}^{2+}\right]_{i}$, the vertical axis is shown as the $340 / 380 \mathrm{~nm}$ light intensity ratio. (a) Vertical scale: 0.10 ; horizontal scale: $20 \mathrm{~s}$, (d) vertical scale: 0.20 ; horizontal scale: $20 \mathrm{s.;}$ (g) vertical scale: 0.10 ; horizontal scale: $20 \mathrm{s.}$, and (j) vertical scale: 0.20; horizontal scale: 20 sec. Panels (b), (e), (h), and (k) display box plots on the absolute area under the curve (AUC) derived from stimulation with the in (a), (d), (g), and (j) mentioned capsaicin-containing solutions. Panels (c), (f), (i), and (l) display box plots with median, 25th and 75th percentiles on the relative AUC of the capsaicin-containing solution-derived $\left[\mathrm{Ca}^{2+}\right]_{i}$ increase and the preceding $20 \mathrm{mM}$ $\mathrm{K}^{+}$-derived $\left[\mathrm{Ca}^{2+}\right]_{i}$ increase. The error bars indicate the 10th and 90th percentiles. Values below the 10th and above the 90 th percentiles are shown as dots. + indicates the mean. ${ }^{*} P<0.05$. 


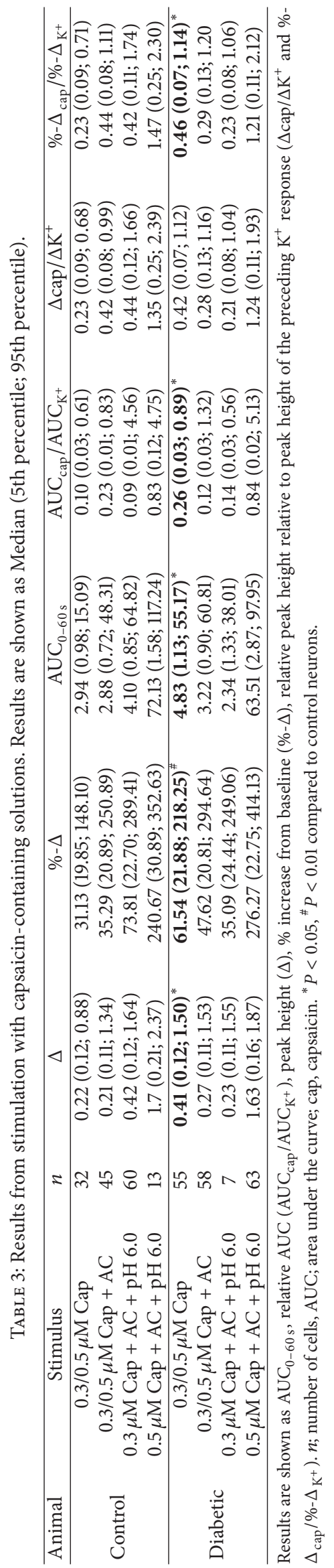




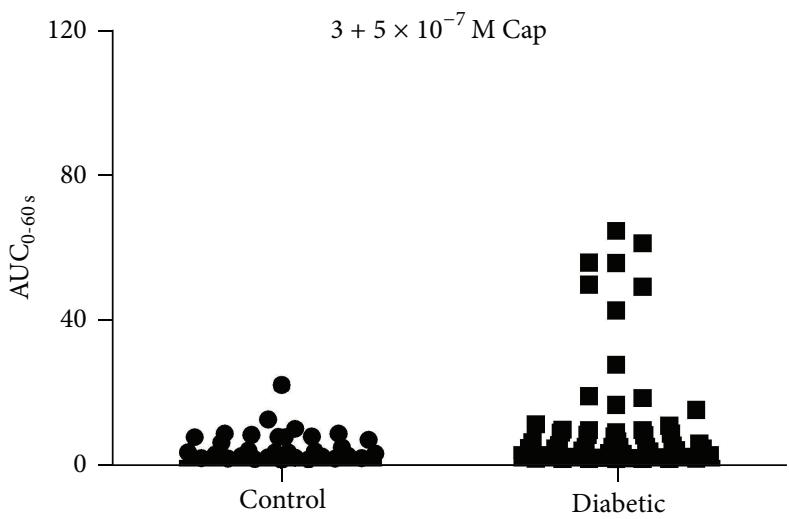

(a)

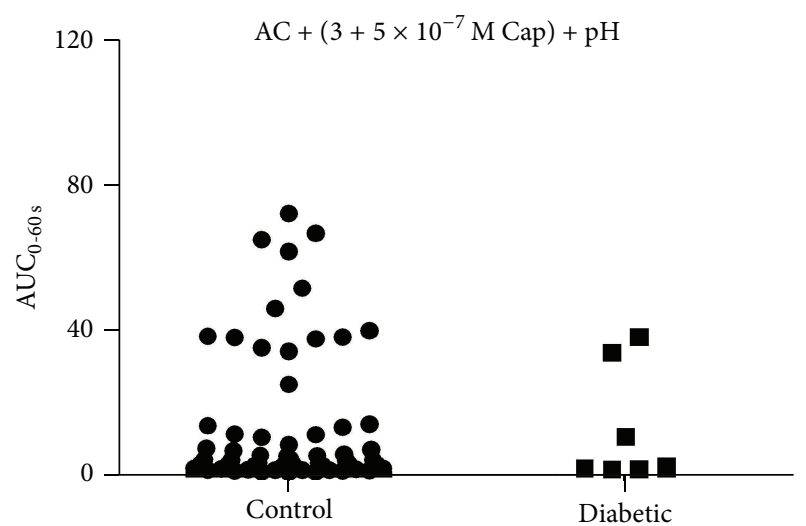

(c)

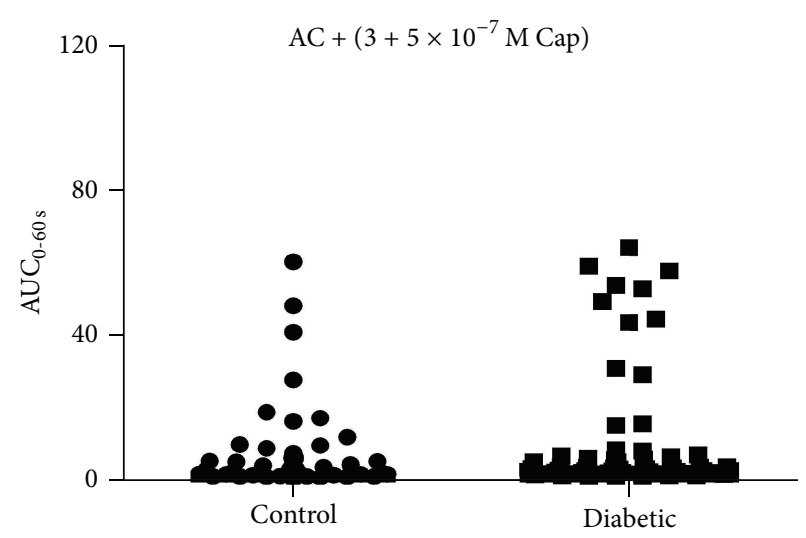

(b)

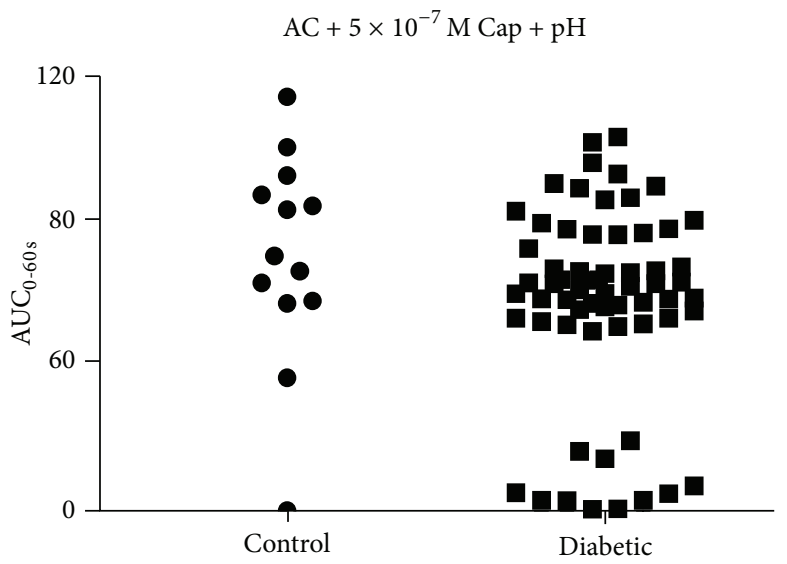

(d)

FIGURE 5: Scatter plots displaying AUC for neurons from control and diabetic animals derived from stimulation with (a) capsaicin, (b) combination of Capsaicin and algogenic chemicals (AC); (c) combination of $0.3 \mu \mathrm{M}$ Capsaicin and AC at pH 6.0, and (d) $0.5 \mu \mathrm{M}$ Capsaicin and $\mathrm{AC}$ at $\mathrm{pH} 6.0$.

duration) [18]. The decrease in amplitude which contrasts the findings of the present study could be explained by the increase in $\left[\mathrm{Ca}^{2+}\right]_{i}$ baseline that Huang et al. found in the DRG neurons from diabetic animals [18], which might have limited the potential change in $\left[\mathrm{Ca}^{2+}\right]_{i}$ in these neurons. The discrepancy between studies might also be related to the duration of diabetes since Huang et al. investigated neurons from STZ-diabetic rats with longer duration (8-14 weeks) of diabetes than what was seen in other studies. The type of diabetes might also influence calcium homeostasis differently, since the present study and the study by Hall et al., both of which found increases in amplitude were performed in $\mathrm{ZDF}$ and $\mathrm{BB} / \mathrm{W}$ rats [32], respectively compared to the other studies in which STZ-diabetic animals were employed $[18,19,29-31,33-35]$.

There is a general agreement on a prolonged recovery following depolarization-induced $\left[\mathrm{Ca}^{2+}\right]_{i}$ currents in neurons from diabetic rats and mice, measured as recovery time, residual $\left[\mathrm{Ca}^{2+}\right]_{i}(60$ or $90 \mathrm{~s}$ after depolarization), or, as in the present study, $\mathrm{AUC}_{(0-180 \mathrm{~s})}[18,19,29-31,34,35]$. This suggests that the diabetes-induced effect on recovery of $\left[\mathrm{Ca}^{2+}\right]_{i}$ is somewhat fundamental as the studies are based on both type 1 and type 2 diabetes, different rodent species, neuron sizes, segmental orientations of the neurons investigated, and on different parts of the nervous system (DRG or DH). The level of $\left[\mathrm{Ca}^{2+}\right]_{i}$ in the DRG neurons is the sum of mechanisms leading to $\mathrm{Ca}^{2+}$-entry into the intracellular space and mechanisms leading to removal of $\mathrm{Ca}^{2+}$ from the intracellular space [36]. During stimulation, intracellular calcium level rises because calcium enters the cell via plasmalemmal voltage- and ligand-gated, and storeoperated $\mathrm{Ca}^{2+}$-channels, and also because calcium is released from the endoplasmatic reticulum [36, 37]. Kinetics of the recovery phase is affected by release of $\mathrm{Ca}^{2+}$ ions from the mitochondria.

Voltage-dependent calcium currents through L- and Nchannels are enhanced in dorsal horn neurons of STZ rats in vitro [33]. This is in accordance with the study by Hall et al. who found a decreased inhibitory effect of the opioid agonist Dyn A in thoracic DRGs from BB/W diabetic rats [32] and an impaired inhibitory G-protein function in thoracic and lumbar DRGs from BB/W and STZ rats, thus contributing to an increased calcium influx [38].

Several mechanisms thus seem to contribute to the increased $\mathrm{K}^{+}$-induced depolarization found in the present 
study, and since these changes are seen in different species, diabetes models, and at different DRG levels, the impact of diabetes on neuronal calcium metabolism seems to be universal.

4.3. Capsaicin Protocols. The present study showed an increased elevation of $\left[\mathrm{Ca}^{2+}\right]_{i}$ following stimulation with capsaicin (pooled results for 0.3 and $0.5 \mu \mathrm{M}$ ) and this agrees with studies by Hall et al. and Hong and Wiley who found an increased peak $\left[\mathrm{Ca}^{2+}\right]_{i}$ in thoracic DRG neurons $(20-40 \mu \mathrm{m})$ from $\mathrm{BB} / \mathrm{W}$ rats and larger and longer-lasting inward currents in DRG neurons from STZ-diabetic rats, respectively, following application of $1 \mu \mathrm{M}$ Capsaicin [32].

The expression of TRPV1 has been shown to be decreased in the hearts of STZ-diabetic mice [39] although Strecker and colleagues reported unaltered capsaicin-induced CGRP release from human right atrium from subjects with diabetes [40].

The findings of increased $\mathrm{Ca}^{2+}$-response to $\mathrm{K}^{+}$and capsaicin show that diabetes causes functional changes in these neurons. The augmented response measured in neurons from diabetic animals following stimulation with capsaicin suggests that the function or expression of TRPV1 might be altered by diabetes. Song et al. have found a decreased expression of membrane TVPR1 in heart [41] which is supported by Hong and Wiley who found decreased TRPV1 expression in small $\mathrm{L}_{1}-\mathrm{L}_{6}$ DRG neurons [42]. They showed, however, in the same study that functional TRPV1 was increased in neurons from diabetic animals and furthermore, the acid-evoked ( $\mathrm{pH}$ 5.0) transient, mediated by TRPV1, was increased in these neurons [42] which is in accordance with the increased capsaicin response seen in the present study.

The function of the PKC-induced phosphorylation, and thus sensitization, of TRPV1 [43] has been shown to be increased in lumbar DRG neurons from STZ diabetic rats [42].

Glutamate receptors (GluR) colocalized with TRPV1 on small diameter DRG neurons have been demonstrated to contribute to the activity of the capsaicin-induced TRPV1 sensitization [44] and GluR could possibly contribute to the finding in the present study that capsaicin-responsive neurons elicited larger $\mathrm{Ca}^{2+}$ responses than neurons from capsaicin-unresponsive neurons (the difference was seen both in diabetic as well as control neurons).

Scatter plots of the responses to capsaicin-containing solutions indicated that the tested neurons might be either high- or low-responders. It cannot be ruled out that diabetes has a differentiated effect on these two potentially different subpopulations of neurons.

When $0.5 \mu \mathrm{M}$ capsaicin was applied in combination with $\mathrm{AC}$ at acidic $\mathrm{pH}$ 6.0, the calcium response was larger (amplitude and AUC) for neurons, regardless of diabetic state, compared to all other applied solutions. This is in accordance with other studies showing that the capsaicininduced neurons activity can be amplified by lowering $\mathrm{pH}$ and by adding inflammatory substances, such as bradykinin $5-\mathrm{HT}, \mathrm{PGE}_{2}$, and adenosine $[10,13,26,27]$. There was no difference, however, between neurons from control and diabetic animals.

\section{Conclusion}

The findings of increased $\mathrm{Ca}^{2+}$-response to $\mathrm{K}^{+}$and capsaicin show that diabetes causes functional changes in the investigated DRG neurons. The augmented response measured in neurons from diabetic animals following stimulation with capsaicin suggests that the function or expression of TRPV1 might be altered which is supported by other studies.

This is, to the best of our knowledge, the first study to investigate calcium activity in DRGs from ZDF rats and the study shows that the diabetes-induced effects on peripheral neuron function have common characteristics with other diabetic animal models. The ZDF rat thus seems to be a relevant model for studying diabetes-induced neuronal alterations in DRG neurons.

Diabetic neuropathy is a very complex condition with multiple factors playing part in the pathophysiology. The present study suggests that diabetes induces changes in DRG neuron function and this might be a contributing factor to the altered pain perception and the altered neuronal response by cardiac neurons seen in diabetic patients during myocardial ischemia and infarction.

\section{Acknowledgments}

This work was supported by The Danish Heart Association, no. 05-04-B120-A476-22223, The Novo Nordisk Foundation (30/11/2006), the Lundbeck Foundation (j.nr. 44/03), and Hørslev-Fonden (2003).

\section{References}

[1] International Diabetes Federation. Diabetes Atlas, 2011, http://www.idf.org/.

[2] J. S. Alpert, S. R. Chipkin, and N. Aronin, "Diabetes mellitus and silent myocardial ischemia," Advances in Cardiology, vol. 37, pp. 297-303, 1990

[3] A. I. Vinik, T. Erbas, M. A. Pfeifer, E. L. Feldman, M. J. Stevens, and J. W. Russell, "Diabetic autonomic neuropathy," in Ellenberg $\&$ Rifkin's Diabetes Mellitus, D. Porte, R. S. Sherwin, and A. Baron, Eds., chapter 46, pp. 789-804, McGraw-Hill, 6th edition, 2003.

[4] C. R. Kahn, G. C. Weir, G. L. King, A. M. Jacobson, A. C. Moses, and R. J. Smith, Eds., Joslin's Diabetes Mellitus, Lippincott Williams \& Wilkins, Philadelphia, Pa, USA, 14th edition, 2005.

[5] A. I. Vinik and D. Ziegler, "Diabetic cardiovascular autonomic neuropathy," Circulation, vol. 115, no. 3, pp. 387-397, 2007.

[6] M. T. Johnstone and G. P. Kinzfogl, "Diabetes and heart disease," in Diabetes and Cardiovascular Disease, M. T. Johnstone and A. Veves, Eds., Contemporary Cardiology, Series edited by C. P.Cannon, chapter 28, pp. 579-628, Humana Press, 2nd edition, 2005.

[7] A. I. Vinik, R. E. Maser, B. D. Mitchell, and R. Freeman, "Diabetic autonomic neuropathy," Diabetes Care, vol. 26, no. 5, pp. 1553-1579, 2003.

[8] P. K. Thomas and D. R. Tomlinson, "Diabetic and hypoglycemic neuropathy," in Peripheral Neuropathy, P. J. Dyck, P. K. Thomas, J. W. Griffin, P. A. Low, and J. F. Poduslo, Eds., chapter 64, pp. 1219-1250, Saunders, 1993.

[9] H. L. Pan, J. C. Longhurst, J. C. Eisenach, and S. R. Chen, "Role of protons in activation of cardiac sympathetic C-fibre afferents 
during ischaemia in cats," Journal of Physiology, vol. 518, no. 3, pp. 857-866, 1999.

[10] S. T. Meller and G. F. Gebhart, "A critical review of the afferent pathways and the potential chemical mediators involved in cardiac pain," Neuroscience, vol. 48, no. 3, pp. 501-524, 1992.

[11] R. D. Foreman, "Mechanisms of cardiac pain," Annual Review of Physiology, vol. 61, pp. 143-167, 1999.

[12] D. C. Kuo, J. J. Oravitz, and W. C. DeGroat, “Tracing of afferent and efferent pathways in the left inferior cardiac nerve of the cat using retrograde and transganglionic transport of horseradish peroxidase," Brain Research, vol. 321, no. 1, pp. 111-118, 1984.

[13] C. J. Benson, S. P. Eckert, and E. W. McCleskey, "Acid-evoked currents in cardiac sensory neurons: a possible mediator of myocardial ischemic sensation," Circulation Research, vol. 84, no. 8, pp. 921-928, 1999.

[14] F. Cervero and J. M. A. Laird, "Visceral pain," The Lancet, vol. 353, no. 9170, pp. 2145-2148, 1999.

[15] F. Cervero, "Sensory innervation of the viscera: peripheral basis of visceral pain,” Physiological Reviews, vol. 74, no. 1, pp. 95-138, 1994.

[16] G. Biessels and W. H. Gispen, "The calcium hypothesis of brain aging and neurodegenerative disorders: significance in diabetic neuropathy," Life Sciences, vol. 59, no. 5-6, pp. 379-387, 1996.

[17] A. Verkhratsky and P. Fernyhough, "Mitochondrial malfunction and $\mathrm{Ca}^{2+}$ dyshomeostasis drive neuronal pathology in diabetes," Cell Calcium, vol. 44, no. 1, pp. 112-122, 2008.

[18] T. J. Huang, N. M. Sayers, P. Fernyhough, and A. Verkhratsky, "Diabetes-induced alterations in calcium homeostasis in sensory neurones of streptozotocin-diabetic rats are restricted to lumbar ganglia and are prevented by neurotrophin-3," Diabetologia, vol. 45, no. 4, pp. 560-570, 2002.

[19] N. V. Voitenko, E. P. Kostyuk, I. A. Kruglikov, and P. G. Kostyuk, "Changes in calcium signalling in dorsal horn neurons in rats with streptozotocin-induced diabetes," Neuroscience, vol. 94, no. 3, pp. 887-890, 1999.

[20] H. D. Schultz, "Cardiac vagal chemosensory afferents: function in pathophysiological states," Annals of the New York Academy of Sciences, vol. 940, pp. 59-73, 2001.

[21] M. L. Ghorbani, C. Qin, M. Wu et al., "Characterization of upper thoracic spinal neurons receiving noxious cardiac and/or somatic inputs in diabetic rats," Autonomic Neuroscience, vol. 165, no. 2, pp. 168-177, 2011.

[22] D. Chen and M. W. Wang, "Development and application of rodent models for type 2 diabetes," Diabetes, Obesity and Metabolism, vol. 7, no. 4, pp. 307-317, 2005.

[23] G. J. Etgen and B. A. Oldham, "Profiling of Zucker diabetic fatty rats in their progression to the overt diabetic state," Metabolism, vol. 49, no. 5, pp. 684-688, 2000.

[24] R. M. Paredes, J. C. Etzler, L. T. Watts, W. Zheng, and J. D. Lechleiter, "Chemical calcium indicators," Methods, vol. 46, no. 3, pp. 143-151, 2008.

[25] D. C. Immke and E. W. McCleskey, "Protons open acid-sensing ion channels by catalyzing relief of $\mathrm{Ca}^{2+}$ blockade," Neuron, vol. 37, no. 1, pp. 75-84, 2003.

[26] M. Kress, S. Fetzer, P. W. Reeh, and L. Vyklicky, "Low pH facilitates capsaicin responses in isolated sensory neurons of the rat," Neuroscience Letters, vol. 211, no. 1, pp. 5-8, 1996.

[27] M. Kress, P. W. Reeh, and L. Vyklicky, "An interaction of inflammatory mediators and protons in small diameter dorsal root ganglion neurons of the rat," Neuroscience Letters, vol. 224, no. 1, pp. 37-40, 1997.
[28] S. G. Lu, X. Zhang, and M. S. Gold, "Intracellular calcium regulation among subpopulations of rat dorsal root ganglion neurons," Journal of Physiology, vol. 577, no. 1, pp. 169-190, 2006.

[29] E. Kostyuk, N. Pronchuk, and A. Shmigol, "Calcium signal prolongation in sensory neurones of mice with experimental diabetes," NeuroReport, vol. 6, no. 7, pp. 1010-1012, 1995.

[30] E. Kostyuk, N. Voitenko, I. Kruglikov et al., "Diabetes-induced changes in calcium homeostasis and the effects of calcium channel blockers in rat and mice nociceptive neurons," Diabetologia, vol. 44, no. 10, pp. 1302-1309, 2001.

[31] A. Shmigol and E. P. Kostyuk, "Mechanisms responsible for calcium signal formation in murine primary sensory neurons: their impairment by experimentally evoked diabetes," Neurophysiology, vol. 27, no. 5, pp. 261-269, 1995.

[32] K. E. Hall, A. A. F. Sima, and J. W. Wiley, "Opiate-mediated inhibition of calcium signaling is decreased in dorsal root ganglion neurons from the diabetic $\mathrm{BB} / \mathrm{W}$ rat," The Journal of Clinical Investigation, vol. 97, no. 5, pp. 1165-1172, 1996.

[33] N. V. Voitenko, I. A. Kruglikov, E. P. Kostyuk, and P. G. Kostyuk, "Effect of streptozotocin-induced diabetes on the activity of calcium channels in rat dorsal horn neurons," Neuroscience, vol. 95, no. 2, pp. 519-524, 1999.

[34] L. Shutov, I. Kruglikov, O. Gryshchenko et al., "The effect of nimodipine on calcium homeostasis and pain sensitivity in diabetic rats," Cellular and Molecular Neurobiology, vol. 26, no. 7-8, pp. 1541-1557, 2006.

[35] E. Kostyuk, N. Svichar, V. Shishkin, and P. Kostyuk, "Role of mitochondrial dysfunction in calcium signalling alterations in dorsal root ganglion neurons of mice with experimentallyinduced diabetes," Neuroscience, vol. 90, no. 2, pp. 535-541, 1999.

[36] M. J. Berridge, M. D. Bootman, and H. L. Roderick, "Calcium signalling: dynamics, homeostasis and remodelling," Nature Reviews Molecular Cell Biology, vol. 4, no. 7, pp. 517-529, 2003.

[37] E. Carafoli, L. Santella, D. Branca, and M. Brini, "Generation, control, and processing of cellular calcium signals," Critical Reviews in Biochemistry and Molecular Biology, vol. 36, no. 2, pp. 107-260, 2001.

[38] K. E. Hall, J. Liu, A. A. F. Sima, and J. W. Wiley, "Impaired inhibitory G-protein function contributes to increased calcium currents in rats with diabetic neuropathy," Journal of Neurophysiology, vol. 86, no. 2, pp. 760-770, 2001.

[39] Z. Wei, L. Wang, J. Han et al., "Decreased expression of transient receptor potential vanilloid 1 impairs the postischemic recovery of diabetic mouse hearts," Circulation Journal, vol. 73, no. 6, pp. 1127-1132, 2009.

[40] T. Strecker, A. Dieterle, P. W. Reeh, M. Weyand, and K. Messlinger, "Stimulated release of calcitonin gene-related peptide from the human right atrium in patients with and without diabetes mellitus," Peptides, vol. 27, no. 12, pp. 3255-3260, 2006.

[41] J. X. Song, L. H. Wang, L. Yao, C. Xu, Z. H. Wei, and L. R. Zheng, "Impaired transient receptor potential vanilloid 1 in streptozotocin-induced diabetic hearts," International Journal of Cardiology, vol. 134, no. 2, pp. 290-292, 2009.

[42] S. Hong and J. W. Wiley, "Early painful diabetic neuropathy is associated with differential changes in the expression and function of vanilloid receptor 1," The Journal of Biological Chemistry, vol. 280, no. 1, pp. 618-627, 2005.

[43] S. Mandadi, P. Armati, and B. D. Roufogalis, "Real-time translocation adn function of PKCbII isoform in response to nociceptive signaling via the TRPV1 pain receptor," Pharmaceuticals, vol. 4, pp. 1503-1517, 2011. 
[44] Y.-H. Jin, M. Takemura, A. Furuyama, and N. Yonehara, "Peripheral glutamate receptors are required for hyperalgesia induced by capsaicin," Pain Research and Treatment, vol. 2012, Article ID 915706, 8 pages, 2012. 


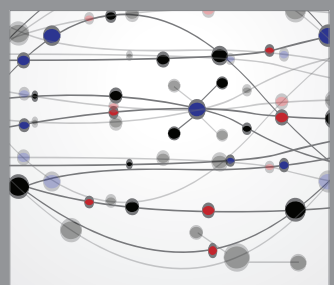

The Scientific World Journal
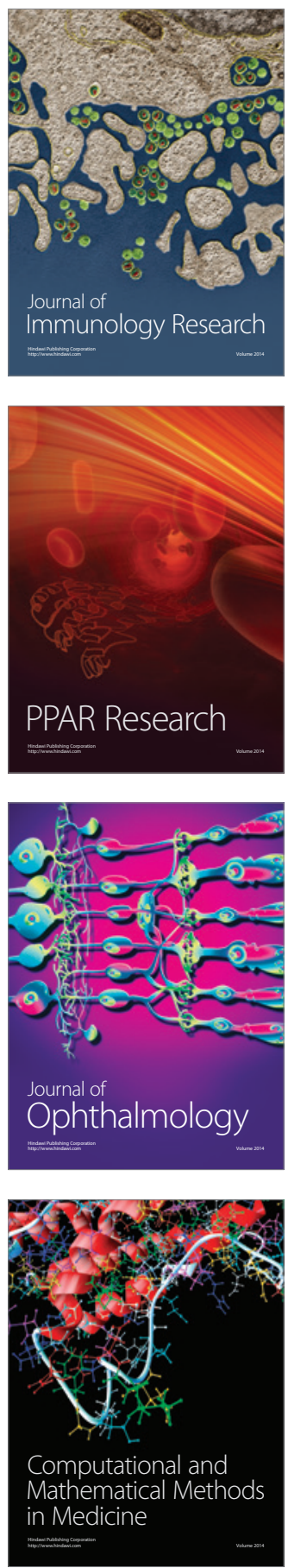

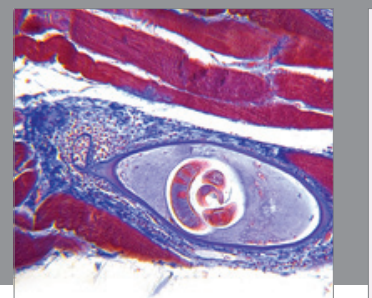

Gastroenterology

Research and Practice
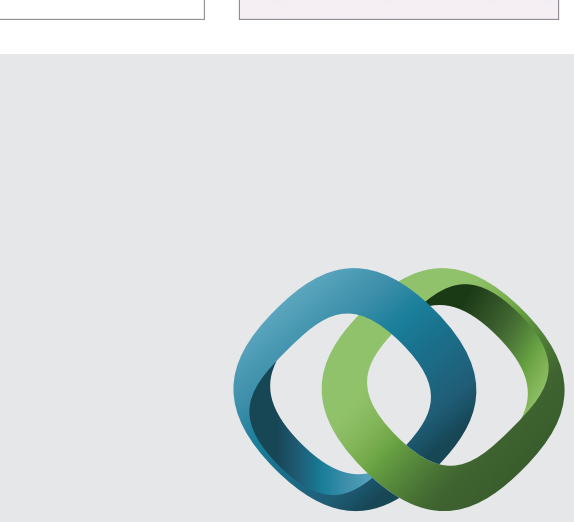

\section{Hindawi}

Submit your manuscripts at

http://www.hindawi.com
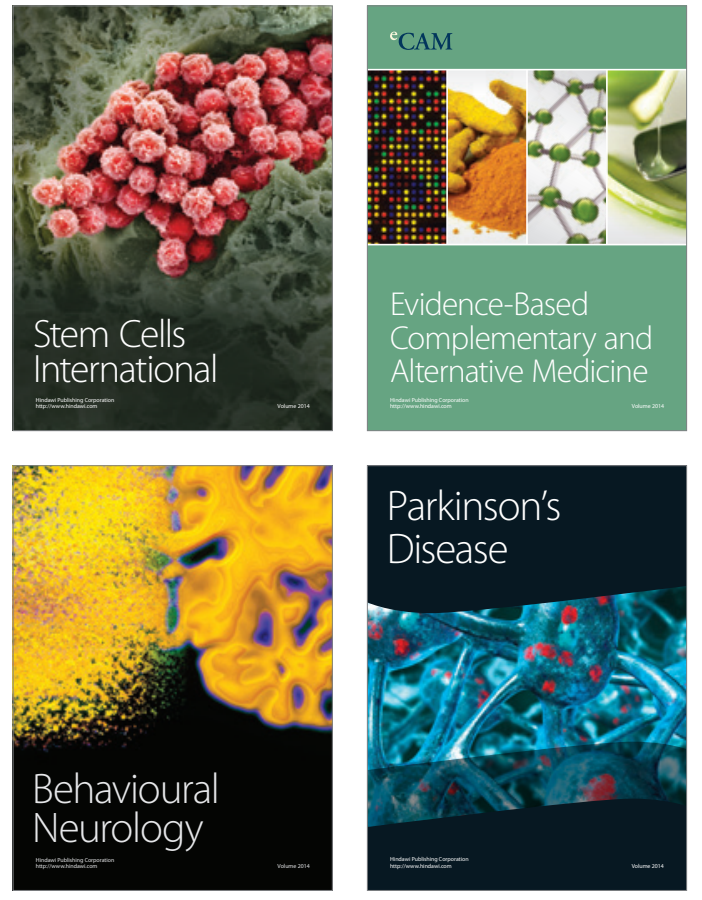
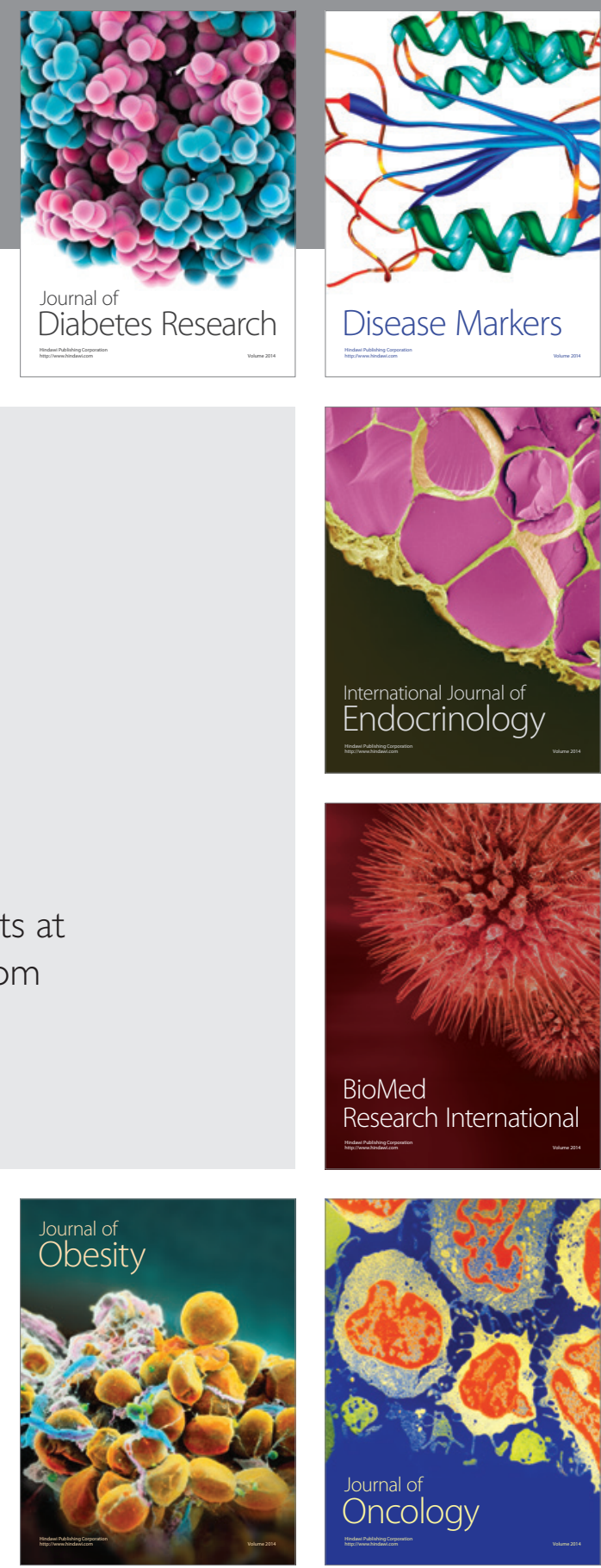

Disease Markers
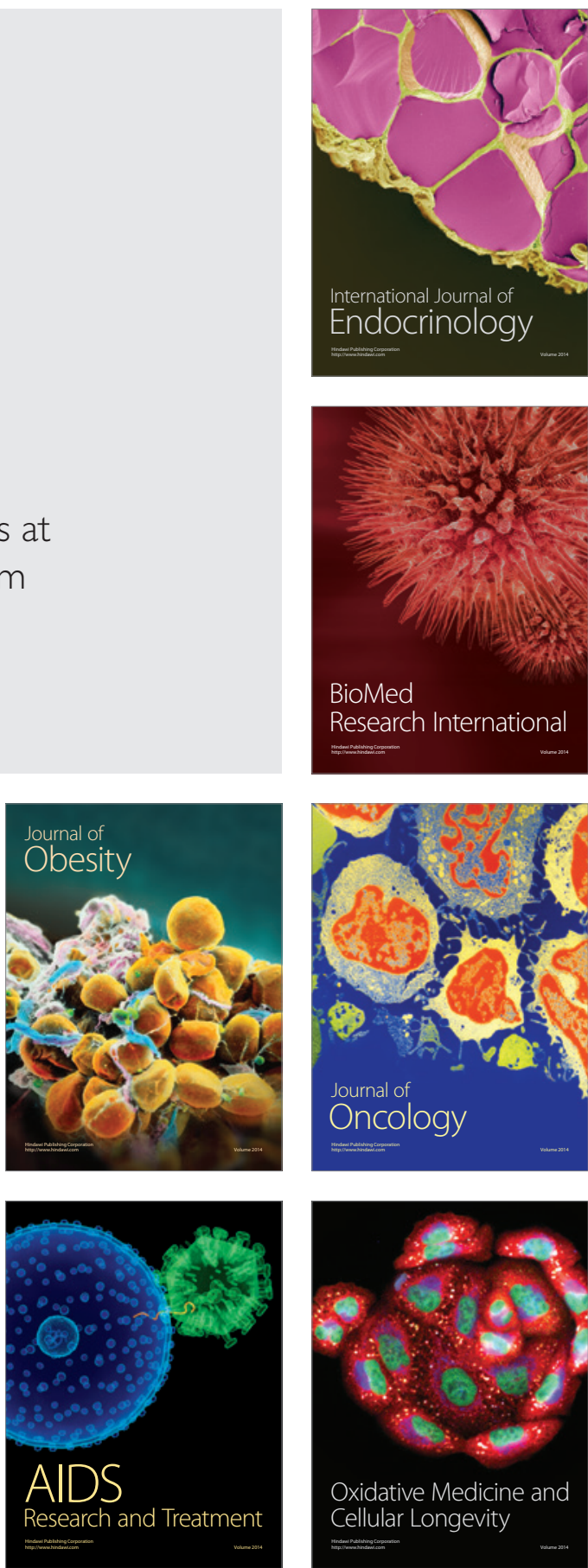